\title{
Exendin-4 attenuates ischemia-induced ventricular arrhythmias in rats
}

\author{
Zhang Kai, Wu Yongbo, Zhao Lin, Guo Jie, Jin Daoqun, Chen Zhiqiang \\ Department of Cardiology, Central Hospital of Huangshi, Huangshi, China
}

\begin{abstract}
Background: Glucagon-like peptide-1 and its receptor agonist-exendin-4 (Ex-4) have been shown to provide beneficial effects for cardiovascular diseases. This study investigated the effects of Ex-4 on ischemia-induced ventricular arrhythmias in rats.

Methods: Anesthetized male rats were once treated with Ex-4 (5 $\mu \mathrm{g} / \mathrm{kg}, \mathrm{i.v}) .1 \mathrm{~h}$ before ischemia in the absence and/or presence of 5-hydroxydecanoic acid (5-HD, $10 \mathrm{mg} / \mathrm{kg}$, i.v., a specific inhibitor of mitochondrial ATP-sensitive potassium [KATP] channels) which were once injected $10 \mathrm{~min}$ before ischemia. And then subjected to ischemia for $30 \mathrm{~min}$. Ventricular arrhythmias were assessed.

Results: During the 30-min ischemia, Ex-4 significantly reduced the incidence of ventricular fibrillation $(V F)(p<0.05)$. The duration of ventricular tachycardia $(V T)+V F$, the number of VT + VF episodes and the severity of arrhythmias were all significantly reduced by Ex-4 compared to those in myocardial ischemia group ( $p<0.05$ for all). Administration of $5-H D$ abolished the protective effects of Ex-4 on VF incidence, the duration of VT $+V F$, the number of VT $+V F$ episodes and the severity of arrhythmias ( $p<0.05$ for all).

Conclusions: This study suggested that Ex-4 could attenuate ischemia-induced ventricular arrhythmias in rats in which mitochondrial KATP channels may be involved. (Cardiol J 2013; 20, 1: 29-33)
\end{abstract}

Key words: exendin-4, ventricular arrhythmias, myocardial ischemia, mitochondrial ATP-sensitive potassium

\section{Introduction}

Glucagon-like peptide-1 (GLP-1), a gut incretin hormone secreted from $\mathrm{L}$ cells in the intestine in response to food intake, has currently been considered as an attractive agent for the management of type-2 diabetes mellitus (T2DM) [1], and the GLP-1 receptor agonist-exendin-4 (Ex-4), which is a 39 amino acid peptide, activates GLP-1 receptors to increase intracellular cAMP in pancreatic acinar cells and has no effect on VIP receptors and function as well as GLP-1. So far, there is emerging evidence that GLP-1 and Ex-4 could provide bene- ficial effects for cardiovascular diseases in both experimental models and patients [2-5]. Recently, Chinda et al. [6] showed that dipeptidyl peptidase-4 inhibitor, a drug for postponing the degradation of GLP-1, could provide an anti-arrhythmic effect during myocardial ischemia (MI), indicating that GLP-1 or GLP-1 receptor agonist may provide an anti-arrhythmic effect during MI.

Mitochondrial ATP-sensitive potassium ( $\left.\mathrm{K}_{\mathrm{ATP}}\right)$ channels have been shown to be a mediator or trigger of ischemic preconditioning or pharmacological preconditioning during MI-reperfusion injury [7-9]. Recently, mitochondrial $\mathrm{K}_{\mathrm{ATP}}$ channels was shown to

Address for correspondence: Chen Zhiqiang, Department of Cardiology, Central Hospital of Huangshi, 43 Wuhan Road, Huangshi, 435000, China, tel: 86-0714-6262061, fax: 86-0714-6262061, e-mail: chenzhiqianghs@163.com

Received: 13.08 .2012

Accepted: 08.10.2012 
be involved in the anti-arrhythmic effects of ischemic preconditioning and pharmacological preconditioning during acute MI [10-12]. Thus, we hypothesized that GLP-1 receptor agonist-Ex-4 may provide an antiarrhythmic effect via mitochondrial $\mathrm{K}_{\text {АтP }}$ channels. This hypothesis was tested in a rat MI model in the present study.

\section{Methods}

Animal preparation and experimental designs

The experimental protocol conformed to the Guideline for the Care and Use of Laboratory Animals published by the US National Institutes of Health (NIH Publication, revised 1996) and was approved by Institutional Animal Care and Use Committee. A total of 40 male Sprague Dawley rats (250-300 g) were randomly assigned into 4 groups receiving the following treatments: Group $1-\mathrm{MI}$ $(n=10)$ : the rats were subjected to left anterior descending coronary artery occlusion for $30 \mathrm{~min}$, $1 \mathrm{~h}$ before coronary artery occlusion, the rats were once treated with sterile saline; Group $2-\mathrm{MI}+$ $+\mathrm{Ex}-4(\mathrm{MI}+\mathrm{Ex}-4)(\mathrm{n}=10)$ : the rats were once treated with Ex-4 (5 $\mu \mathrm{g} / \mathrm{kg}$, i.v., Sigma, USA) [13] $1 \mathrm{~h}$ before left anterior descending coronary artery occlusion. The Ex- 4 was dissolved in sterile saline; Group 3-MI + Ex-4 + 5-hydroxydecanoic acid (MI-Ex-4-5-HD) $(\mathrm{n}=10)$ : after rats were treated with Ex-4, 5-HD (a specific of mitochondrial $\mathrm{K}_{\mathrm{ATP}}$ channels inhibitor, $10 \mathrm{mg} / \mathrm{kg}$, i.v., Sigma, USA) [11] was once injected $10 \mathrm{~min}$ before left anterior descending coronary artery occlusion. The 5-HD was dissolved in sterile saline; Group $4-\mathrm{MI}+5$-HD (MI-5-HD) $(\mathrm{n}=10): 5-\mathrm{HD}(10 \mathrm{mg} / \mathrm{kg}$, i.v. $)$ was once injected $10 \mathrm{~min}$ before left anterior descending coronary artery occlusion.

After being anesthetized with sodium pentobarbital (45 mg/kg, i.p.), the rats were ventilated artificially with a volume-controlled rodent respirator at 70 strokes per minute. All rats were placed on an electric heating pad to maintain their body temperature at $37^{\circ} \mathrm{C}$. Heparin (200 IU/kg, i.v.) was given before ischemia. The right common carotid artery and left jugular vein were cannulated to measure mean arterial blood pressure (MBP) and administrate saline or drugs, respectively. Lead II of the electrocardiogram was monitored with subcutaneous stainless steel electrodes. A computerbased EP system (LEAD2000B, Jinjiang Ltd, China) was used for recording MBP, heart rate and electrocardiogram.

A thoracotomy through a left parasternal incision was performed. The pericardium was incised,

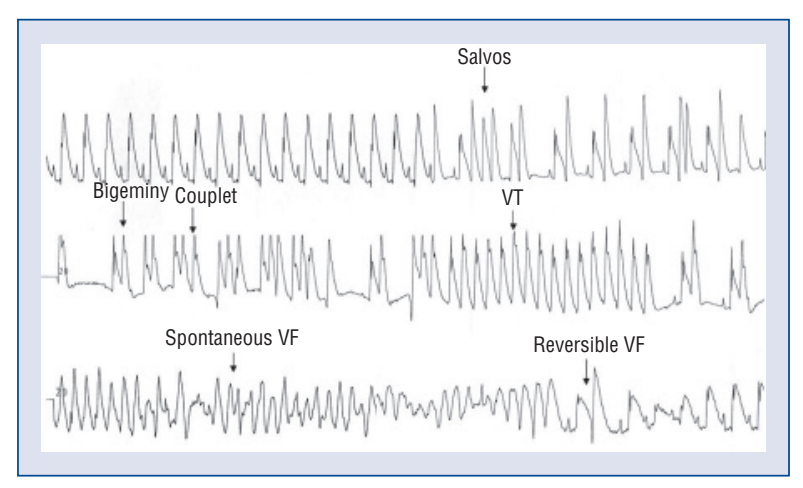

Figure 1. Representative examples of different ventricular arrhythmias during the 30-min ischemia; VT ventricular tachycardia; VF — ventricular fibrillation.

and the anterior wall of the left ventricle was exposed. A 4-0 silk suture on a small curved needle was passed through the myocardium beneath the middle segment of the left anterior descending coronary artery occlusion branch crossing down the middle of the anterior wall of the left ventricle. A successful MI model was confirmed by ST segment elevation in leads II and regional cyanosis of the myocardial surface.

\section{Assessment of ventricular arrhythmias}

Ischemia-induced ventricular arrhythmias were determined in accordance with the Lambeth Conventions [11, 12]. Ventricular ectopic beat (VEB) was defined as identifiable premature QRS complexes (premature with respect to the P wave). Ventricular tachycardia (VT) was defined as the occurrence of four or more consecutive VEBs at a rate faster than the resting sinus rate. Ventricular fibrillation (VF) was defined as unidentifiable and low voltage QRS complexes. Other patterns of VEBs such as bigeminy, couplet (two consecutive VEBs) and salvos (three consecutive VEBs) were included in VEBs and not analyzed separately. VF may be sustained or may convert spontaneously to a normal sinus rhythm. VF resulting in death was considered as irreversible (Fig. 1).

The severity of arrhythmias was quantified by the following scoring system $[11,12]$ :

- 0: 0-50 VEBs with no other arrhythmias during the $30-\mathrm{min}$ ischemia period,

- 1: only 50-500 VEBs,

- 2: more than $500 \mathrm{VEBs}$, or one episode of spontaneously reversible VT or VF,

- 3: 2-30 episodes of spontaneously reversible VT and/or VF, 
Table 1. Heart rate and MBP before and after 30-min ischemia.

\begin{tabular}{|c|c|c|c|c|c|}
\hline \multirow[t]{2}{*}{ Group } & \multirow[t]{2}{*}{$\mathbf{N}$} & \multicolumn{2}{|c|}{ Heart rate [bmp] } & \multicolumn{2}{|c|}{ MBP [mm Hg] } \\
\hline & & Before ligation & After ligation & Before ligation & After ligation \\
\hline MI & 10 & $395 \pm 16$ & $390 \pm 15$ & $98 \pm 7$ & $92 \pm 9$ \\
\hline MI-Ex-4 & 10 & $396 \pm 17$ & $390 \pm 14$ & $97 \pm 6$ & $90 \pm 11$ \\
\hline MI-Ex-4-5-HD & 10 & $397 \pm 14$ & $392 \pm 20$ & $95 \pm 9$ & $90 \pm 13$ \\
\hline MI-5-HD & 10 & $398 \pm 18$ & $390 \pm 21$ & $97 \pm 10$ & $92 \pm 11$ \\
\hline
\end{tabular}

Data were expressed as mean \pm SD. There were no significant differences in heart rate and MBP among each group. Heart rate and MBP after $30-m i n$ ligation were lower than those before 30-min ligation, but no statistical significance were found (all $\mathrm{p}>0.05$ ); $\mathrm{Ml}-\mathrm{myocardial}$ ischemia; Ex-4 - exendin-4; 5-HD - 5-hydroxydecanoic acid; MBP — mean arterial blood pressure

- 4: more than 30 episodes of spontaneously reversible VT and/or VF,

- 5: irreversible VF.

\section{Statistical analysis}

All values were expressed as mean $\pm \mathrm{SD}$ or median (interquartile range) for continuous variables or the percentage of incidence of ventricular arrhythmia. One-way ANOVA or Welch was used for comparisons among groups and the StudentNeuman-Keuls or Dunnett T3 was used for posthoc multiple comparisons. Paired samples t test was used for comparisons of heart rate and MBP within each group. Mann-Whitney U test was used for arrhythmia score between two groups; when more than two groups were compared, Kruskale-Wallis $\mathrm{H}$ test was used. Incidences of ventricular arrhythmias (i.e. VT and VF) were used for comparisons by Fisher's exact test. Statistical significance was defined as $\mathrm{p}<0.05$.

\section{Results}

\section{Hemodynamics}

There were no significant differences in heart rate and MBP among each group ( $p>0.05$ for all). Heart rate and MBP after 30-min ligation were slightly lower than those before 30-min ligation, but no statistical significances were found ( $p>0.05$ for all; Table 1 ).

\section{Ventricular arrhythmias during ischemia}

In this MI model, severe ventricular arrhythmias peaked at 5-25 min after coronary artery ligation. Figure 1 shows different ventricular arrhythmias during the 30 -min ischemia.

\section{Incidences of VT and VF}

The incidence of VF $(1 / 10,10.0 \%)$ in MI-Ex-4 group was significantly decreased compared to that

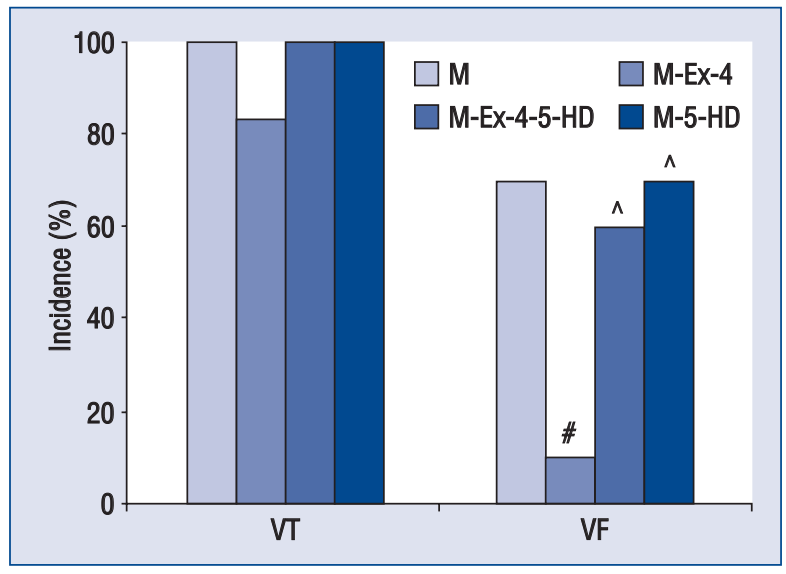

Figure 2. The incidences of VT and VF in MI, MI-Ex-4, Ml-Ex-4-5-HD and Ml-5-HD group ( $n=10$ for each group); $\# p<0.05$ vs. Ml group; ^ $p<0.05$ vs. Ml-Ex-4 group; abbreviations as in Table 1 and Figure 1.

in MI group (7/10, 70.0\%; $\mathrm{p}<0.05)$; however, there was no significant difference in VT incidence $(9 / 10$, $90.0 \%$ vs. $10 / 10,100 \%$ in MI group; $p>0.05$ ). Administration of 5-HD could abolish the protective effect of Ex-4 on VF (6/10, 60.0\%; p < 0.05). 5-HD alone did not affect the incidences of VT and VF during acute MI compared to those in MI group, respectively ( $p>0.05$ for all; Fig. 2).

\section{Durations of VT + VF}

The duration of VT + VF in MI-Ex-4 group $(24.8 \pm 10.5 \mathrm{~s})$ was significantly shorter than that in MI group $(78.4 \pm 21.9 \mathrm{~s} ; \mathrm{p}<0.05)$. Administration of 5-HD abolished the effects of Ex-4 on duration of VT + VF $(71.3 \pm 19.7 \mathrm{~s})$ compared to that in MI-Ex-4 group ( $<0.05)$. 5-HD alone did not affect the duration of VT + VF during acute MI compared to that in MI group, respectively ( $>$ > 0.05; Fig. 3). 


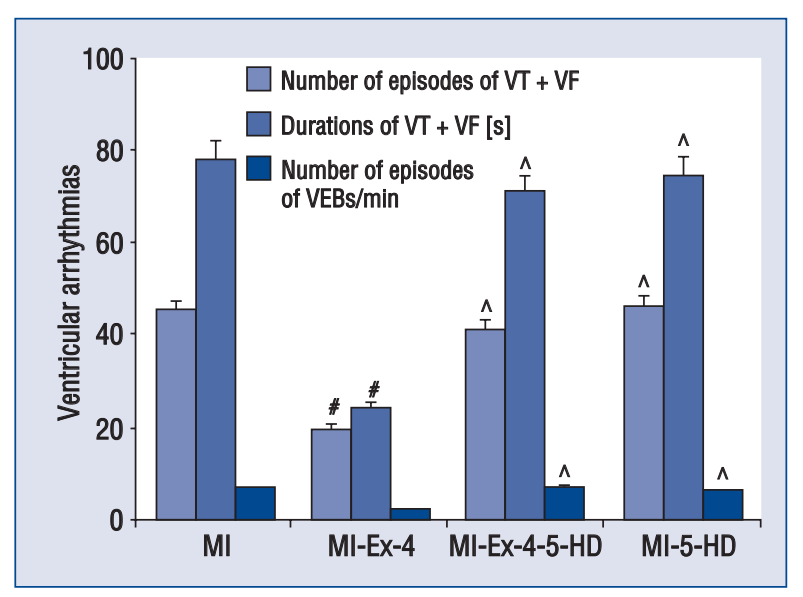

Figure 3. Durations of episodes of VF + VT and VEBs/ /min during 30-min ischemia in Ml, Ml-Ex-4, Ml-Ex-4-5-HD and MI-5-HD group ( $\mathrm{n}=10$ for each group); \#p < $<0.05$ vs. Ml group; ^ $\mathrm{p}<0.05$ vs. MI-Ex-4 group; VEBs - ventricular ectopic beats; other abbreviations as in Table 1 and Figure 1.

\section{Number of episodes of VF + VT and VEBs/min \\ The number of episodes of VT $+\mathrm{VF}$ and VEBs/ $/ \mathrm{min}$ in MI-Ex-4 group $(19.8 \pm 9.8$ and $2.2 \pm 0.9)$ were significantly decreased compared to those in MI group (45.5 \pm 11.3 and $6.9 \pm 1.9$; $\mathrm{p}<0.05)$. Ad- ministration of 5 -HD could abolish the protective effects of Ex-4 on the number of episodes of VT + $+\mathrm{VF}(41.4 \pm 7.9)$ and $\mathrm{VEBs} / \mathrm{min}(7.0 \pm 2.1)$ compared to those in MI-Ex-4 group ( $\mathrm{p}<0.05$ for both). 5 -HD alone did not affect the number of episodes of VT + $+\mathrm{VF}$ and $\mathrm{VEBs} /$ min during acute MI compared to those in MI group, respectively ( $p>0.05$ for both; Fig. 3 ).}

\section{Severity of arrhythmias}

The severity of ventricular arrhythmias was significantly attenuated by Ex-4 (3.00 [median], 2.25-3.00 [interquartile range]) compared to that in MI group (4.00, 4.00-4.00) $(\mathrm{p}<0.05)$. Administration of 5-HD (4.00, 4.00-4.00) abolished the anti-arrhythmic effect of Ex-4 on the severity of ventricular arrhythmias compared to that in MI-Ex-4 ( $<<0.05)$. 5 -HD alone did not affect the severity of ventricular arrhythmias during acute MI compared to that in MI group, respectively (both $\mathrm{p}>0.05$; Fig. 4).

\section{Discussion}

In this study, we found that Ex-4 could attenuate ischemia-induced ventricular arrhythmias in rats. Meanwhile, 5-HD, a specific inhibitor of mitochondrial $\mathrm{K}_{\mathrm{ATP}}$ channels could abolish the anti-arrhythmic effect of Ex-4. Thus, we speculated that Ex-4 could

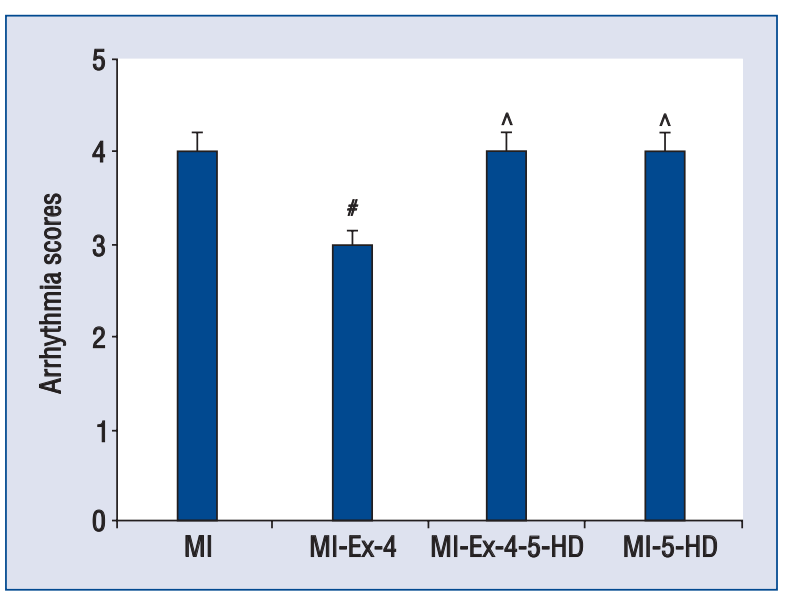

Figure 4. Distribution of the arrhythmia score during 30-min ischemia in MI, MI-Ex-4, Ml-Ex-4-5-HD and MI-5 -HD group ( $n=10$ for each group); \#p $<0.05$ vs. Ml group; $\wedge p<0.05$ vs. MI-Ex-4 group; abbreviations as in Table 1 and Figure 1.

attenuate ischemia-induced ventricular arrhythmias by mediating mitochondrial $\mathrm{K}_{\text {ATP }}$ channels.

Previous studies showed that GLP-1 and GLP-1 receptor agonists could reduce MI-reperfusion injury and improve cardiac function $[2-5,14]$. Recently, Chinda et al. [6] firstly showed that dipeptidyl peptidase-4 inhibitor, a drug for postponing the degradation of GLP-1 and could promote GLP-1 to exert more longer protective effect during MI, could attenuated the shortening of the effective refractory period, decreased the number of ventricular premature beats, increased the VF threshold, suggesting that GLP-1 could decrease ischemia-induced ventricular arrhythmias. In the present study, we found that Ex-4, a GLP-1 receptor agonist, could also attenuate the genesis of ventricular arrhythmias during MI without affecting hemodynamics. Yamamoto et al. [15] showed that GLP-1 receptor stimulation including Ex-4 could increase blood pressure and heart rate in a dose dependent, however, these affection only last little time and could return to basal levels within $40-50 \mathrm{~min}$. In this study, we inject Ex- $41 \mathrm{~h}$ before ischemia, thus, Ex-4 did not affect the hemodynamics during MI period. These further indicated that GLP-1 and its receptor agonists may provide an anti-arrhythmic effect during MI.

Mitochondrial $\mathrm{K}_{\mathrm{ATP}}$ channels was involved in the anti-arrhythmic effect of ischemic preconditioning or pharmacological preconditioning during MI [7-9]. In this study, we found that 5-HD, a specific inhibitor of mitochondrial $\mathrm{K}_{\mathrm{ATP}}$ channels could abo- 
lish the anti-arrhythmic effect of Ex-4, indicating that mitochondrial $\mathrm{K}_{\mathrm{ATP}}$ channels may be involved in the anti-arrhythmic effect of Ex-4. Many studies showed that activation of mitochondrial $\mathrm{K}_{\mathrm{ATP}}$ channels could limit mitochondrial $\mathrm{Ca}^{2+}$ overload and maintain mitochondrial $\mathrm{Ca}^{2+}$ homeostasis during $\mathrm{MI}$ $[16,17]$; mitochondrial $\mathrm{Ca}^{2+}$ homeostasis plays a fundamental role in cytosolic free $\mathrm{Ca}^{2+}$ homeostasis and could prevent ischemia-induced cytosolic free $\mathrm{Ca}^{2+}$ overload $[18,19]$ which plays an important role in ischemia-induced ventricular arrhythmias [20]. Thus, mitochondrial $\mathrm{K}_{\mathrm{ATP}}$ channels was involved in the anti-arrhythmic effect of Ex-4 during MI.

\section{Limitations of the study}

In the study, we only investigated the effect of Ex-4 on ischemia-induced ventricular arrhythmias and observed that the specific inhibitor of mitochondrial $\mathrm{K}_{\mathrm{ATP}}$ channels could abolish the anti-arrhythmic effect of Ex-4. The precise mechanisms underlying our observation and their clinical relevance will require future elucidation. We need to investigate the effect of Ex-4 the change of intracellular $\mathrm{Ca}^{2+}$ concentration or $\mathrm{Ca}^{2+}$ channels in the future study.

\section{Conclusions}

The present study suggested that Ex-4 could attenuate ischemia-induced ventricular arrhythmias in rats which may be through mediating mitochondrial $\mathrm{K}_{\mathrm{ATP}}$ channels.

The study was supported by the Subject of Health Department of Huangshi City of China (No. 2012-139).

Conflict of interest: none declared

\section{References}

1. Kieffer TJ, Habener JF. The glucagon-like peptides. Endocr Rev, 1999; 20: 876-913.

2. Nikolaidis LA, Elahi D, Hentosz T et al. Recombinant glucagon-like peptide-1 increases myocardial glucose uptake and improves left ventricular performance in conscious dogs with pacing-induced dilated cardiomyopathy. Circulation, 2004; 110: 955-961.

3. Sokos GG, Nikolaidis LA, Mankad S et al. Glucagon-like peptide-1 infusion improves left ventricular ejection fraction and functional status in patients with chronic heart failure. J Card Fail, 2006; 12: 694-699.
4. Sonne DP, Engstrom T, Treiman M. Protective effects of GLP-1 analogues exendin-4 and GLP-1(9-36) amide against ischemiareperfusion injury in rat heart. Regul Pept, 2008; 146: 243-249.

5. Okerson T, Chilton RJ. The cardiovascular effects of GLP-1 receptor agonists. Cardiovasc Ther, 2012; 30: e146-e155.

6. Chinda K, Palee S, Surinkaew S et al. Cardioprotective effect of dipeptidyl peptidase-4 inhibitor during ischemia-reperfusion injury. Int J Cardiol, 2012; doi:10.1016/j.ijcard.2012.01.011.

7. Gross GJ. The role of mitochondrial K-ATP channels in cardioprotection. Basic Res Cardiol, 2000; 95: 280-284.

8. Gross GJ, Fryer RM. Mitochondrial K-ATP channels: Triggers or distal effecters of ischemic or pharmacological preconditioning? Circ Res, 2000; 87: 431-433.

9. Gourine AV, Molosh AI, Poputnikov D et al. Endothelin-1 exerts a preconditioning-like cardioprotective effect against ischaemia/ /reperfusion injury via the ETA receptor and the mitochondrial K-ATP channelss in the rat in vivo. Br J Pharmacol, 2005; 144: 331-337.

10. Vegh A, Parratt JR. The role of mitochondrial K-ATP channels in antiarrhythmic effects of ischaemic preconditioning in dogs. Br J Pharmacol, 2002; 137: 1107-1115.

11. Imani A, Faghihi M, Sadr SS et al. Noradrenaline reduces ischemia-induced arrhythmia in anesthetized rats: Involvement of alpha(1)-adrenoceptors and mitochondrial K-ATP channels. J Cardiovasc Electrophysiol, 2008; 19: 309-315.

12. $\mathrm{Hu} X, \mathrm{Wu} B$, Wang $\mathrm{X}$ et al. Minocycline attenuates ischemia-induced ventricular arrhythmias in rats. Eur J Pharmacol, 2011; 654: 274-279.

13. Wu L, Olverling A, Huang $Z$ et al. GLP-1, exendin-4 and $\mathrm{C}$-peptide regulate pancreatic islet microcirculation, insulin secretion and glucose tolerance in rats. Clin Sci, 2012; 122: 375-384.

14. Timmers L, Henriques JPS, de Kleijn DPV et al. Exenatide reduces infarct size and improves cardiac function in a porcine model of ischemia and reperfusion injury. J Am Coll Cardiol, 2009; 53: 501-510.

15. Yamamoto H, Lee CE, Marcus JN et al. Glucagon-like peptide-1 receptor stimulation increases blood pressure and heart rate and activates autonomic regulatory neurons. J Clin Invest, 2002; 110: $43-52$.

16. Holmuhamedov EL, Wang L, Terzic A. ATP-sensitive K+ channels openers prevent $\mathrm{Ca} 2+$ overload in rat cardiac mitochondrial. J Physiol, 1999; 519: 347-360.

17. Wang L, Cherednichenko G, Hernandez L et al. Preconditioning limits mitochondrial $\mathrm{Ca}(2+)$ during ischemia in rat hearts: Role of K(ATP) channels. Am J Physiol Heart Circ Physiol, 2001; 280: H2321-2328.

18. Murata M, Akao M, O’Rourke et al. Mitochondrial ATP-sensitive potassium channels attenuate matrix $\mathrm{Ca}(2+)$ overload during simulated ischemia and reperfusion: Possible mechanism of cardioprotection. Circ Res, 2001; 89: 891-898.

19. Gao H, Chen L, Yang HT. Activation of alpha ${ }_{18}$-adrenoceptors alleviates ischemia/reperfusion injury by limitation of mitochondrial Ca2+ overload in cardiomyocytes. Cardiovasc Res, 2007; 75: 584-595.

20. Clusin WT. Calcium and cardiac arrhythmias: DADs, EADs, and alterns. Crit Rev Clin Lab Sci, 2003; 40: 337-375. 